\title{
Transesophageal echocardiography
} for cardiovascular risk estimation in patients with sepsis and new-onset atrial fibrillation: a multicenter prospective pilot study

Vincent Labbé ${ }^{1,2^{*}}$, Stephane Ederhy ${ }^{3,4}$, Nathanael Lapidus ${ }^{5,6}$, Jérémie Joffre ${ }^{7}$, Keyvan Razazi ${ }^{2,8}$, Laurent Laine ${ }^{9}$, Oumar Sy ${ }^{10}$, Sebastian Voicu ${ }^{11}$, Frank Chemouni ${ }^{12}$, Nadia Aissaoui ${ }^{13}$, Roland Smonig ${ }^{14}$, Denis Doyen ${ }^{15}$, Fabrice Carrat ${ }^{5,6}$, Guillaume Voiriot ${ }^{1,2}$, Armand Mekontso-Dessap ${ }^{2,8}$, Ariel Cohen ${ }^{3,4,16}$ and Muriel Fartoukh ${ }^{1,2}$ on behalf of for the FAST Study Group

\begin{abstract}
Background: Echocardiographic parameters have been poorly investigated for estimating cardiovascular risk in patients with sepsis and new-onset atrial fibrillation. We aim to assess the prevalence of transesophageal echocardiographic abnormalities and their relationship with cardiovascular events in mechanically ventilated patients with sepsis and new-onset atrial fibrillation.

Methods: In this prospective multicenter pilot study, left atrial/left atrial appendage (LA/LAA) dysfunction, severe aortic atheroma, and left ventricular systolic dysfunction were assessed using an initial transesophageal echocardiographic study, which was repeated after $48-72 \mathrm{~h}$ to detect LA/LAA thrombus formation. The study outcome was a composite of cardiovascular events at day 28 , including arterial thromboembolic events (ischemic stroke, non-cerebrovascular arterial thromboembolism, LA/LAA thrombus), major bleeding, and all-cause death.
\end{abstract}

Results: The study population comprised 94 patients (septic shock 63\%; 35\% women; median age 69 years). LA/LAA dysfunction, severe aortic atheroma, and left ventricular systolic dysfunction were detected in 17 (19\%), 22 (24\%), and 27 (29\%) patients, respectively. At day 28, the incidence of cardiovascular events was $46 \%$ (95\% confidence interval [CI]: 35 to 56). Arterial thromboembolic events and major bleeding occurred in 7 (7\%) patients (5 ischemic strokes, 1 non-cerebrovascular arterial thromboembolism, 2 left atrial appendage thrombi) and 18 (19\%) patients, respectively. At day 28, 27 patients (29\%) died. Septic shock (hazard ratio [HR]: 2.36; 95\% Cl 1.06 to 5.29) and left ventricular systolic dysfunction (HR: 2.06; 95\% Cl 1.05 to 4.05) were independently associated with cardiovascular events.

Conclusions: Transesophageal echocardiographic abnormalities are common in mechanically ventilated patients with sepsis and new-onset atrial fibrillation, but only left ventricular systolic dysfunction was associated with cardiovascular events at day 28.

Keywords: Atrial fibrillation, Transesophageal echocardiography, Stroke, Bleeding, Sepsis

*Correspondence: vincent.labbe@aphp.fr

${ }^{1}$ Sorbonne Université, Assistance Publique-Hôpitaux de Paris

(AP-HP), Service de Médecine Intensive Réanimation, Département Médico-Universitaire APPROCHES, Hôpital Tenon, 4 rue de la Chine,

75020 Paris, France

Full list of author information is available at the end of the article

\section{Background}

New-onset atrial fibrillation (NOAF) is the commonest arrhythmia in the intensive care unit (ICU) occurring in one-third of critically ill patients with sepsis $[1$, 2]. In this setting, patients with NOAF are at greater risk 
of arterial thromboembolic events [2] and death than patients without NOAF [1, 2]. Decision-making with regard to thrombo-prophylaxis should be based upon the absolute risks of cardiovascular events, including arterial thromboembolism event, bleeding, and death, and the net clinical benefit for a given patient. However, conventional score to assess thromboembolic and bleeding risk in patient with atrial fibrillation have not been validated in the specific setting of sepsis $[3,4]$.

Transesophageal echocardiography (TEE) abnormalities, such as left ventricular (LV) systolic dysfunction, left atrial/left atrial appendage (LA/LAA) dysfunction, and severe aortic atheroma, are associated with an increased risk of arterial thromboembolic event in patients with atrial fibrillation [5]. In particular, LA/LAA dysfunction, revealed by LAA low velocities and dense spontaneous echo contrast (SEC) resulting from blood stasis [6-8], remains strongly associated with thrombus formation [9]. So, bedside echocardiography could be useful in critically ill patients with sepsis and NOAF for estimating their cardiovascular risk.

We therefore conducted a prospective, multicenter, observational, echocardiographic pilot study (the Fibrillation Atrial Sepsis Thrombus [FAST] study) in 10 French ICUs in patients with sepsis and NOAF. Our objectives were to assess early LV systolic dysfunction, LA/LAA dysfunction, and severe aortic atheroma, and to investigate the relationship of these structural and functional parameters with the occurrence of cardiovascular events at day 28.

\section{Methods}

\section{Patient selection}

During a 24-month period (November 2014 to November 2016), all mechanically ventilated adult patients with sepsis/septic shock who experienced significant NOAF (including atrial fibrillation and atrial flutter) on ICU admission or during their ICU stay were eligible. Significant NOAF was defined by an AF in patients with no prior history of AF [10], lasting at least $6 \mathrm{~h}$ or recurred more than twice (>30 s) per day despite correction of modifiable risk factors such as hypokalemia or hypovolemia [11]. Sepsis/septic shock was defined according to the Sepsis-3 definition [12]. All episodes of NOAF were systematically recorded on an electrocardiogram assessed by two cardiologists (any discrepancy being solved by consensus), and classified as atrial fibrillation, or atrial flutter using standard definitions [13]. Patients with a history of atrial fibrillation for which the cardiovascular risk depends in part to their established previous medication regimen for atrial fibrillation [10], with valvular heart disease classifying AF as "valvular AF" (significant mitral stenosis, mechanical aortic or mitral valve; [14]), with TEE contraindication [15], those who were moribund (expected survival $<48 \mathrm{~h}$ ), or with a decision to limit full care, and those refusing to participate, were not included.

The study was approved by the Comité de Protection des Personnes Ile-de-France 5 (ref. 14941), as a component of standard care, and patient consent was waived, as per French Law. Written and oral information about the study was given to the patients or their next of kin.

\section{Data collection}

Demographics, medical history, antithrombotic medications, admission category, Simplified Acute Physiology Score II [16], and $\mathrm{CHA}_{2} \mathrm{DS}_{2}$-VASc [14] and HAS-BLED [14] scores were recorded on ICU admission (detailed definitions in Additional file 1: Table S1). The type of infection and the Sepsis-Related Organ Failure assessment score [17] were recorded at NOAF onset (day 0). NOAF management during ICU stay was left at the discretion of the physicians in charge.

\section{Echocardiography}

Echocardiography were performed at the bedside by skilled intensivists, all of whom had $\geq 2$ years of TEE experience, with competence in advanced critical care echocardiography [18]. Examinations were conducted with recent commercially available equipment (CX50, AFFINITY 70, and IE33; Philips Ultrasound system, Bothell, WA; VIVID 7, VIVID 9, and VIVID I, General Electric Healthcare system, Horten, Norway). All echocardiographic studies involved transthoracic echocardiography according to the recommendations of the European Association of Cardiovascular Imaging [19] with 2.5- and 3-MHz transducers, followed by TEE with $5-\mathrm{MHz}$ multiplane transducers. Each patient underwent echocardiography within $48 \mathrm{~h}$ of NOAF onset (initial TEE). Based on the initial TEE findings, changes in treatment regarding therapeutic anticoagulation were left at the discretion of the physicians in charge. To investigate LA/LAA thrombus formation, a second echocardiography was performed 48 to $72 \mathrm{~h}$ after the initial TEE in patients who were still mechanically ventilated (second TEE). Supplementary echocardiography studies performed at the discretion of the physicians in charge were recorded.

LA or LAA thrombi, LA/LAA dysfunction (including LA/LAA dense SEC and LAA low velocities), LAA large area, and severe aortic atheroma were investigated as previously described $[6,7,20-24]$. A LA/LAA thrombus was considered present when there was a well-circumscribed, echodense, intra-cavitary mass that is acoustically distinct from the underlying endocardium and the pectinate muscles [21]. LA/LAA dense SEC was defined 
as finely reticular pattern of dynamic, swirling intra-cavitary echoes localized within the LA or LAA persistent throughout the LA-LAA at normal gain [6, 21]. Velocity of the LAA was recorded with pulse-wave Doppler interrogation $1 \mathrm{~cm}$ within the orifice. Flow velocity was evaluated in anterograde (emptying) and retrograde (filling) directions, and was averaged over a minimum of 3 to 5 consecutive cardiac cycles as specified by protocol. LAA low velocity was defined as a LAA filling or emptying velocity $<25 \mathrm{~cm} / \mathrm{s}[7,23]$. The area of the LAA was measured by planimetry at $90^{\circ}$ view as specified by protocol. LAA large area was defined as a LAA area $>5 \mathrm{~cm}^{2}$ [21]. The thoracic aorta was analyzed at each level, including the ascending aorta, the proximal and distal arch, and the descending aorta, as specified by protocol and according to previously described methods [20]. Severe aortic atheroma was defined as: protruding atherosclerotic plaques (highly echogenic areas that protruded $\geq 4 \mathrm{~mm}$ above the surface of the intima into the aortic lumen); or mobile atheroma; or ulcerated atheroma [20, 22, 24]. LV systolic dysfunction was defined as LV ejection fraction $\leq 45 \%$ (mild, 31-45\%; severe, $\leq 30 \%$ ) using the biplane Simpson method [19].

All echocardiographic studies were analyzed off-line by two experienced observers (VL and SE) in a blinded manner. Differences between observers were resolved by consensus; if the observers could not agree, a third observer (AC) reviewed the studies, and that observer's judgment was binding.

\section{Follow-up and outcomes}

All patients were followed from day 0 (inclusion) to day 28. The primary outcome was the occurrence of a cardiovascular event, comprising arterial thromboembolic events (ischemic stroke, non-cerebrovascular thromboembolism, or thrombus of the LA/LAA on echocardiography studies [3]), major bleeding event (according to the International Society on Thrombosis and Hemostasis definition: symptomatic bleeding in a critical area or organ such as intracranial, bleeding associated with a reduction in hemoglobin of $\geq 1.24 \mathrm{mmol} / \mathrm{L}$ or leading to transfusion of $\geq 2$ units blood or packed cells, or fatal bleeding; [25, 26]), and death from any cause. Secondary outcomes were individual components of the primary outcome (detailed definitions in Additional file 1: Table S1).

With the exception of the second TEE, no systematic screening of arterial thromboembolic events was performed. The diagnostic work-up of arterial thromboembolic events and major bleeding events included the usual investigations performed in the participating ICUs, and was collected by the attending intensivists. An independent committee adjudicated arterial thromboembolic events and major bleeding events, and assessed the relationship of ischemic stroke and non-cerebrovascular thromboembolism with NOAF, using the Stop Stroke Study Trial of Org 10172 in Acute Stroke Treatment classification (detailed definitions in Additional file 1: Table S2, Fig. S1, [27]).

\section{Statistical analysis}

Data are reported as medians (interquartile range [IQR]) for quantitative variables, and as frequencies (percentages) for categorical variables. Associations with composite primary outcome (cardiovascular events) were tested by standard Cox models. A multivariable model for the primary outcome was built from studied TEE parameters (LV systolic dysfunction, LA/LAA dysfunction, severe aortic atheroma), with additional adjustment on variables associated with this outcome in the univariate analysis. Associations with secondary outcomes (individual component of the composite primary outcome) were tested by univariate cause-specific Cox models for the first occurrence of arterial thromboembolic event or major bleeding event (accounting for the competing risk of death), and by standard Cox models for the all-cause mortality. A multivariable model was built for the primary outcome only, as the number of events was judged too low to avoid overfitting for the other outcomes. All survival models were censored at day 28 . Hazard ratios (HRs) were estimated and reported with their 95\% confidence intervals (CIs). All tests were 2-tailed and $p$ values $<0.05$ were considered significant. Statistical analysis was conducted with R version 3.6.3 (R Core Team 2019; $\mathrm{R}$ foundation for statistical Computing, Vienna, Austria).

\section{Results}

\section{Baseline clinical characteristics and TEE findings}

During the study period, 94 patients were studied (63 men and 31 women) with a median age of 69 years (IQR: 61 to 77 years) (study flowchart in Fig. 1). Median time from ICU admission to NOAF onset was 2 days (IQR: 1 to 4 days) (atrial fibrillation, $n=93$; atrial flutter, $n=1)$. Baseline clinical characteristics are displayed in Table 1. Median $\mathrm{CHA}_{2} \mathrm{DS}_{2}$-VASc score was 3 (IQR: 2 to 4 ), and HAS-BLED score was 2 (IQR: 1 to 3). Initial TEE studies were performed after a median time of 1.3 days (IQR: 0.7 to 2.1 days) from NOAF onset. NOAF was present during initial TEE in $47 \%$ of patients (details regarding other hemodynamic parameters during TEE are available in Additional file 1: Table S3). LA/LAA dysfunction was detected in $19 \%$ of patients, including LA/LAA SEC (7\%) and LAA low velocities (12\%). Severe aortic atheroma was diagnosed in $24 \%$ of patients. LV systolic dysfunction occurred in $29 \%$ of patients. None of the patients had thrombus in the LA 
385 patients aged $\geq 18$ years with mechanical ventilation, sepsis, and significant atrial fibrillation

\section{Excluded $(n=268)$}

History of atrial fibrillation $(n=210)$

Contraindication to TEE $(n=18)$

Moribund $(n=18)$

History of valvular heart disease $(n=11)$

Decision to limit full care $(n=10)$

Refused to participate $(n=1)$

Non-inclusion $(n=21)$

No research staff available in time $(n=13)$

No affiliation to social security $(n=5)$

Inadvertent omission from the study inclusion process $(n=3)$

\section{Secondary excluded $(n=2)$}

Patient with failed attempts to introduce the TEE probe

Patient with no sepsis

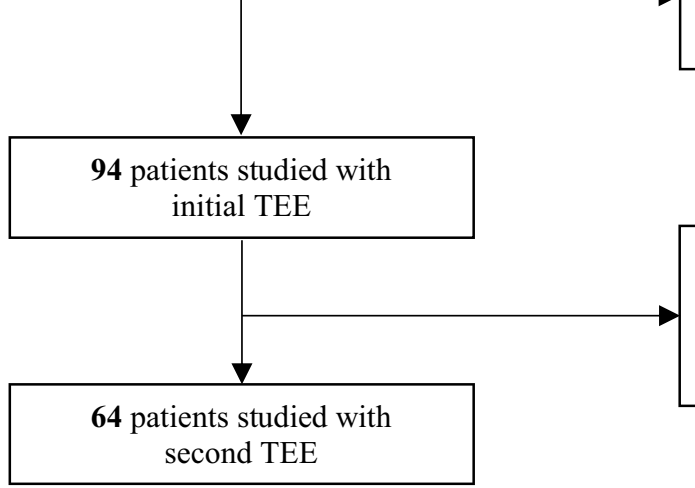

\section{No second TEE $(\mathbf{n}=30)$}

Weaning of invasive mechanical ventilation $(n=15)$

Organizational reasons $(\mathrm{n}=7)$

Death $(n=8)$

Fig. 1 Patient flowchart. TEE transesophageal echocardiography

or LAA (Table 2). Regarding the therapeutic impact of TEE studies, therapeutic anticoagulation was initiated following TEE in $3 \%$ of patients due to LA/LAA dense SEC $(n=2)$ and severe aortic atheroma $(n=1)$.

\section{NOAF management during ICU stay}

Cardioversion was attempted in 67 patients (71\%), using amiodarone in 65 patients (69\%) and electric shock in 23 patients (24\%). Management prior to electric shock included therapeutic anticoagulation in 8 of 23 patients and TEE to exclude LA/LAA thrombus in 13 of 23 patients. During ICU stay, therapeutic anticoagulation was administered in 50 patients (53\%), after 1 day (IQR: 0 to 2 days) from NOAF onset. At day 28, NOAF was persistent in 15 patients (16\%) (Additional file 1: Table S4).

\section{Outcomes}

The incidence of cardiovascular events at day 28 was 46\% (95\% CI: 35 to 56), and 27 patients (29\%) died. 7 patients $(7 \%)$ presented at least one arterial thromboembolic event occurring after a median of 6 days (IQR: 4 to 7 days) from NOAF onset. Arterial thromboembolic events included 5 ischemic strokes (three definitely, one probably, and one definitely not related to NOAF), 1 noncerebral thromboembolism (probably related to NOAF), and 2 LAA thrombi. A second TEE was performed in 64 patients (Fig. 1) after 4.2 days (IQR: 3.3 to 5.1 days) from NOAF onset, and revealed 1 LAA thrombus. The second LAA thrombus was diagnosed in a further TEE, performed 17 days after NOAF onset because of the clinical occurrence of ischemic stroke. Details about the mechanisms of each arterial thromboembolic event are shown 
Table 1 Baseline clinical characteristics, initial severity, and initial new-onset atrial fibrillation management according to 28-day arterial thromboembolic events, major bleeding events and death

\begin{tabular}{|c|c|c|c|c|c|c|c|}
\hline \multirow[t]{2}{*}{ Variable } & \multirow[t]{2}{*}{ Total $(n=94)$} & \multicolumn{2}{|c|}{$\begin{array}{l}\text { Arterial thromboembolic } \\
\text { event }\end{array}$} & \multicolumn{2}{|c|}{ Major bleeding event } & \multicolumn{2}{|l|}{ Death } \\
\hline & & No $(n=87)$ & Yes $(n=7)$ & No $(n=76)$ & Yes $(n=18)$ & No $(n=67)$ & Yes $(n=27)$ \\
\hline \multicolumn{8}{|l|}{ Baseline clinical characteristics on ICU admission } \\
\hline Age, median (IQR), years & $69(61-77)$ & $69(61-77)$ & $69(63-80)$ & $69(61-78)$ & $67(61-74)$ & $69(61-77)$ & $68(64-78)$ \\
\hline Female sex, no. (\%) & $33(35)$ & $31(36)$ & $2(29)$ & $26(34)$ & $7(39)$ & $25(37)$ & $8(30)$ \\
\hline Cardiac disease, no. (\%) & $17(18)$ & $14(16)$ & $3(43)$ & $14(18)$ & $3(17)$ & $13(19)$ & $4(15)$ \\
\hline Left ventricular systolic dysfunction, no. (\%) & $3(3)$ & $3(3)$ & 0 & $3(4)$ & 0 & $3(4)$ & 0 \\
\hline Vascular disease, no. (\%) & $19(20)$ & $16(18)$ & $3(43)$ & $14(18)$ & $5(28)$ & $11(16)$ & $8(30)$ \\
\hline Stroke, no. (\%) & $8(8)$ & $8(9)$ & 0 & $7(9)$ & $1(6)$ & $5(7)$ & $3(11)$ \\
\hline Diabetes mellitus, no. (\%) & $24(25)$ & $23(26)$ & $1(14)$ & $21(28)$ & $3(17)$ & $14(21)$ & $10(37)$ \\
\hline Smoker, no. (\%) & $51(54)$ & $48(55)$ & $3(43)$ & $42(55)$ & $9(50)$ & $37(55)$ & $14(52)$ \\
\hline Hypertension, no. (\%) & $57(61)$ & $51(59)$ & $6(86)$ & $46(60)$ & $11(61)$ & $38(57)$ & $19(70)$ \\
\hline \multicolumn{8}{|l|}{ Clinical risk scores, median (IQR) } \\
\hline $\mathrm{CHA}_{2} \mathrm{DS}_{2}-\mathrm{VASC}$ & $3(2-4)$ & $3(2-4)$ & $3(1-4)$ & $3(2-4)$ & $3(2-4)$ & $3(2-4)$ & $3(2-4)$ \\
\hline HAS-BLED & $2(1-3)$ & $2(1-3)$ & $2(1-3)$ & $2(1-3)$ & $2(1-3)$ & $2(1-3)$ & $2(1-3)$ \\
\hline Previous therapeutic anticoagulation, no. (\%) & $4(4)$ & $4(5)$ & 0 & $2(3)$ & $2(11)$ & $4(6)$ & 0 \\
\hline \multicolumn{8}{|l|}{ Admission category, no. (\%) } \\
\hline Medical & $74(79)$ & $70(80)$ & $4(57)$ & $61(80)$ & $13(72)$ & $52(78)$ & $22(81)$ \\
\hline Scheduled surgery & $5(5)$ & $5(6)$ & 0 & $4(5)$ & $1(6)$ & $4(6)$ & $1(4)$ \\
\hline Emergency surgery & $15(16)$ & $12(14)$ & $3(43)^{\mathrm{a}}$ & $11(14)$ & $4(22)$ & $11(16)$ & $4(15)$ \\
\hline SAPS II score on ICU admission, median (IQR) & $58(47-74)$ & $58(44-74)$ & $63(51-68)$ & $60(44-74)$ & $54(50-73)$ & $54(43-73)$ & $65(55-74)$ \\
\hline \multicolumn{8}{|c|}{ Severity and management on the day of NOAF onset } \\
\hline SOFA score, median (IQR) & $9(7-11)$ & $9(7-11)$ & $9(6-9)$ & $9(7-11)$ & $9(6-11)$ & $9(7-11)$ & $10(6-13)$ \\
\hline Septic shock, no. (\%) & $59(63)$ & $55(63)$ & $4(57)$ & $45(59)$ & $14(78)$ & $38(57)$ & $21(78)$ \\
\hline Catecholamine, no. (\%) & $75(80)$ & $70(80)$ & $5(71)$ & $60(79)$ & $15(83)$ & $52(78)$ & $23(85)$ \\
\hline Norepinephrine & $71(75)$ & $66(76)$ & $5(71)$ & $57(75)$ & $14(78)$ & $50(75)$ & $21(78)$ \\
\hline Epinephrine & $6(6)$ & $5(6)$ & $1(14)$ & $3(4)$ & $3(17)^{b}$ & $4(6)$ & $2(7)$ \\
\hline Dobutamine & $7(7)$ & $5(6)$ & $2(29)$ & $7(9)$ & 0 & $5(7)$ & $2(7)$ \\
\hline Cardioversion attempt, no. (\%) & $60(64)$ & $58(67)$ & $2(29)$ & $49(64)$ & $11(61)$ & $42(63)$ & $18(67)$ \\
\hline Medical (amiodarone) & $59(63)$ & $57(65)$ & $2(29)$ & $48(63)$ & $11(61)$ & $41(61)$ & $18(67)$ \\
\hline Electrical & $16(17)$ & $16(18)$ & 0 & $9(12)$ & $7(39)^{b}$ & $10(15)$ & $6(22)$ \\
\hline Antiplatelet therapy, no. (\%) & $24(25)$ & $21(24)$ & $3(43)$ & $17(22)$ & $7(39)$ & $15(22)$ & $9(33)$ \\
\hline Therapeutic anticoagulation, no. (\%) & $28(30)$ & $25(29)$ & $3(43)$ & $23(30)$ & $5(28)$ & $23(34)$ & $5(18)$ \\
\hline
\end{tabular}

$\mathrm{CHA}_{2} \mathrm{DS}_{2}$-VASC congestive heart failure, hypertension, age $\geq 75$ years (doubled), diabetes mellitus, prior stroke or transient ischemic attack or thromboembolism (doubled), vascular disease, age 65 to 74 years, sex category (female), HAS-BLED hypertension, abnormal renal/liver function, stroke, bleeding history or predisposition, labile international normalized ratio, elderly, drugs/alcohol concomitantly, ICU intensive care unit, IQR interquartile, NOAF new-onset atrial fibrillation, SAPS Simplified Acute Physiology Score, SOFA Sepsis-Related Organ Failure Assessment

a $p<0.05$, when compared with the value for no arterial thromboembolic event

${ }^{\mathrm{b}} p<0.05$, when compared with the value for no major bleeding event

in Table 3. 18 patients (19\%) had at least one major bleeding event occurring after a median of 8.5 days (IQR: 3.5 to 12 days) from NOAF onset. Major bleeding events included 21 extra-cranial and 2 intracranial bleedings (Additional file 1: Table S5). 10 patients (11\%) presented a major bleeding event categorized as life-threatening, including fatal gastrointestinal bleeding in 2 of them. At the time of the first arterial thromboembolic and major bleeding events, therapeutic anticoagulation was used in 5 patients (71\%) and 12 patients (67\%), respectively.

\section{Factors associated with cardiovascular events}

Baseline clinical characteristics were similar between patients with or without cardiovascular event (Additional file 1: Table S6). Among initial severity and NOAF management, septic shock and electrical cardioversion attempt on the day of NOAF onset were associated with cardiovascular events (respectively, HR: 2.72; 95\% CI 1.30 to 5.69 and HR: 2.33; 95\% CI 1.17 to 4.65 ; Additional file 1: Table S6). Regarding TEE parameters, 
Table 2 Initial transthoracic and transesophageal echocardiographic variables according to 28-day arterial thromboembolic events, major bleeding events, and death

\begin{tabular}{|c|c|c|c|c|c|c|c|}
\hline \multirow[t]{2}{*}{ Variable } & \multirow[t]{2}{*}{ Total $(n=94)$} & \multicolumn{2}{|c|}{$\begin{array}{l}\text { Arterial } \\
\text { thromboembolic event }\end{array}$} & \multicolumn{2}{|c|}{ Major bleeding event } & \multicolumn{2}{|l|}{ Death } \\
\hline & & No $(n=87)$ & Yes $(n=7)$ & No $(n=76)$ & Yes $(n=18)$ & No $(n=67)$ & Yes $(n=27)$ \\
\hline LVEF, median (IQR), \% ( $n=94)$ & $55(45-61)$ & $56(45-62)$ & $51(33-57)$ & $57(49-62)$ & $47(43-57)$ & $57(49-61)$ & $51(30-61)^{c}$ \\
\hline $\begin{array}{l}\text { LV systolic dysfunction (LVEF } \leq 45 \%) \text {, no. (\%) } \\
(n=94)\end{array}$ & $27(29)$ & $24(28)$ & $3(43)$ & $18(23)$ & $9(50)^{b}$ & $16(24)$ & $11(41)$ \\
\hline $\begin{array}{l}\text { LV systolic severe dysfunction (LVEF } \leq 30 \%) \text {, no. (\%) } \\
(n=94)\end{array}$ & $9(10)$ & $7(8)$ & $2(29)$ & $9(12)$ & 0 & $2(3)$ & $7(26)^{c}$ \\
\hline RV dilatation, no. $(\%)(n=90)^{d}$ & $27(28)$ & $25(30)$ & $2(29)$ & $22(30)$ & $5(29)$ & $21(33)$ & $6(23)$ \\
\hline Paradoxical septum, no. (\%) $(n=94)$ & $5(5)$ & $4(5)$ & $1(14)$ & $5(5)$ & 0 & $3(4)$ & $2(7)$ \\
\hline Significant left-sided valve disease, no. (\%) $(n=94)^{\mathrm{e}}$ & $4(4)$ & $2(2)$ & $2(29)^{a}$ & $1(1)$ & $3(17)^{b}$ & $2(3)$ & $2(7)$ \\
\hline LA area, median (IQR), $\mathrm{cm}^{2}(n=70)$ & $20(16-24)$ & $20(16-23)$ & $25(18-28)$ & $20(16-23)$ & $21(18-24)$ & $21(16-25)$ & $18(15-22)$ \\
\hline LA/LAA thrombus, no. $(\%)(n=94)$ & 0 & 0 & 0 & 0 & 0 & 0 & 0 \\
\hline LAA dysfunction, no. (\%) $(n=88)^{f}$ & $17(19)$ & $16(20)$ & $1(14)$ & $12(17)$ & $5(28)$ & $12(19)$ & $5(19)$ \\
\hline LA/LAA dense SEC $(n=94)$ & $7(7)$ & $6(7)$ & $1(14)$ & $6(8)$ & $1(6)$ & $5(7)$ & $2(7)$ \\
\hline LAA low velocity $(n=88)^{9}$ & $11(12)$ & $11(14)$ & 0 & $7(10)$ & $4(22)$ & $7(11)$ & $4(15)$ \\
\hline LAA emptying velocity, median (IQR), $\mathrm{cm} / \mathrm{s}(n=89)$ & $66(41-85)$ & $67(41-83)$ & $63(41-90)$ & $66(43-81)$ & $65(33-91)$ & $61(44-85)$ & $70(39-81)$ \\
\hline LAA filling velocity, median (IQR), $\mathrm{cm} / \mathrm{s}(n=88)$ & $60(46-71)$ & $59(45-71)$ & $65(50-68)$ & $62(46-72)$ & $56(45-61)$ & $58(47-72)$ & $62(44-68)$ \\
\hline LAA large area $\left(>5 \mathrm{~cm}^{2}\right)$, no. (\%) $(n=87)$ & $13(15)$ & $10(12)$ & $3(43)$ & $10(14)$ & $3(18)$ & $7(11)$ & $6(24)$ \\
\hline LAA area, median (IQR), $\mathrm{cm}^{2}(n=87)$ & $3.2(2.4-4.3)$ & $3.2(2.4-4.1)$ & $4.9(3.0-5.3)$ & $3.2(2.4-4.3)$ & $3.4(2.3-4.5)$ & $3.4(2.3-4.2)$ & $3.1(2.4-4.5)$ \\
\hline Severe aortic atheroma, no. (\%) $(n=91)$ & $22(24)$ & $21(25)$ & $1(14)$ & $18(24)$ & $4(23)$ & $18(27)$ & $4(16)$ \\
\hline
\end{tabular}

IQR interquartile, $L A$ left atrial, $L A A$ left atrial appendage, $L V$ left ventricular, $L V E F$ left ventricular ejection fraction, $R V$ right ventricular, $S E C$ spontaneous echo contrast

${ }^{a} p<0.05$, when compared with the value for no arterial thromboembolic event

${ }^{\mathrm{b}} p<0.05$, when compared with the value for no major bleeding event

${ }^{c} p<0.05$, when compared with no death

${ }^{\mathrm{d}} \mathrm{RV}$ dilatation was defined as $\mathrm{RV}$ and $\mathrm{LV}$ end-diastolic areas ratio in long-axis cardiac view

${ }^{\mathrm{e}}$ Aortic stenosis, $n=2$; mitral regurgitation, $n=1$; aortic regurgitation, $n=1$

${ }^{f}$ LA/LAA dense SEC or LAA low velocity

${ }^{g}$ LAA emptying velocity $<25 \mathrm{~cm} / \mathrm{s}$ or LAA filling velocity $<25 \mathrm{~cm} / \mathrm{s}$

only LV systolic dysfunction was associated with cardiovascular events (HR: 2.75; 95\% CI 1.48 to 5.08; Additional file 1: Table S3). As compared with patients showing no LV systolic dysfunction, patients with mild LV systolic dysfunction had a higher cardiovascular risk (HR: $2.39 ; 95 \%$ CI 1.14 to 4.63 ) and those with severe LV systolic dysfunction had an even higher risk (HR: 4.2; $95 \%$ CI 1.79 to 10.05 ). A multivariable model built from TEE parameters with additional adjustment on septic shock and electrical cardioversion attempt on the day of NOAF onset identified LV systolic dysfunction (HR: 2.06; 95\% CI 1.05 to 4.05 ) as the only independent predictor of cardiovascular events (Table 4). Additional adjustments on baseline clinical characteristics (SAPSII score, CHADS2Vasc2 and HAS-BLED) and antithrombotic management (antiplatelet therapy and therapeutic anticoagulation on the day of NOAF onset) did not substantially change the magnitude of reported hazard ratios (Additional file 1: Table S7).

\section{Factors associated with each of the components of cardiovascular events Arterial thromboembolic event}

There was no significant difference in baseline clinical characteristics, severity, and management on the day of NOAF onset between patients with and without arterial thromboembolic event, with the exception of a context of emergency surgery (Table 1). In particular, the $\mathrm{CHA}_{2} \mathrm{DS}_{2}$-VASc score was similar between the two groups. Regarding TEE parameters, only significant leftsided valve disease was associated with an increased risk of arterial thromboembolic event (Table 2).

\section{Major bleeding event}

Patients with major bleeding event more often received initial electrical cardioversion attempt, whereas the HAS-BLED score and initial therapeutic anticoagulation (Table 1) were similar between the two groups. LV systolic dysfunction and left-sided valve disease were 
Table 3 Description of 28-day arterial thromboembolic events

\begin{tabular}{|c|c|c|c|c|c|c|c|}
\hline Variable & Patient 1 & Patient 2 & Patient 3 & Patient 4 & Patient 5 & Patient 6 & Patient 7 \\
\hline $\mathrm{CHA}_{2} \mathrm{DS}_{2}$-VASc score & 3 & 6 & 0 & 1 & 2 & 5 & 4 \\
\hline NOAF characteristics & Persistent & Sustained & Sustained & Sustained & Persistent & Sustained & Persistent \\
\hline $\begin{array}{l}\text { Time from NOAF onset, } \\
\text { days }\end{array}$ & 11 & 2 & 6 & 5 & 5 & 0 & 3 \\
\hline \multicolumn{8}{|l|}{ Initial TEE study } \\
\hline $\begin{array}{l}\text { LV systolic function, } \\
\text { LVEF (\%) }\end{array}$ & 45 & 50 & 5 & 56 & 70 & 60 & 22 \\
\hline LA/LAA dysfunction ${ }^{\text {a }}$ & No & No & No & No & No & No & Yes \\
\hline Severe aortic atheroma & Yes & No & No & No & No & No & No \\
\hline \multicolumn{8}{|c|}{ Management before arterial thromboembolic event } \\
\hline $\begin{array}{l}\text { Therapeutic anticoagu- } \\
\text { lation }\end{array}$ & Yes & No & Yes & Yes & Yes & No & Yes \\
\hline Cardioversion attempt & Yes & Yes & Yes & Yes & No & No & No \\
\hline Type & Ischemic stroke & Ischemic stroke & Ischemic stroke & Ischemic stroke & $\begin{array}{l}\text { Ischemic stroke; LAA } \\
\text { thrombus }^{\text {b }}\end{array}$ & Non-CVTE & LAA thrombus ${ }^{c}$ \\
\hline \multicolumn{8}{|l|}{ Mechanism ${ }^{d}$} \\
\hline $\begin{array}{l}\text { Causatives subtypes of } \\
\text { ischemic stroke }\end{array}$ & $\begin{array}{l}\text { Probable large artery } \\
\text { atherosclerosis }\end{array}$ & $\begin{array}{l}\text { Evident cardio- } \\
\text { aortic embolism }\end{array}$ & $\begin{array}{l}\text { Evident cardio- } \\
\text { aortic embolism }\end{array}$ & $\begin{array}{l}\text { Evident cardio- } \\
\text { aortic embolism }\end{array}$ & $\begin{array}{l}\text { Evident cardio-aortic } \\
\text { embolism }\end{array}$ & - & - \\
\hline $\begin{array}{l}\text { Sources of embolism } \\
\text { other than intracardiac } \\
\text { thrombus }^{f}\end{array}$ & - & No & VA ECMO & No & No & $\begin{array}{l}\text { Suspicion of } \\
\text { endocarditis }\end{array}$ & - \\
\hline Relation to NOAF & Definitely not & Definitely & Probably & Definitely & Definitely & Probably & - \\
\hline $\begin{array}{l}\text { Fatal ischemic stroke or } \\
\text { non-CVTE }\end{array}$ & No & No & No & No & No & No & - \\
\hline
\end{tabular}

$C H A_{2} D S_{2}$-VASC congestive heart failure, hypertension, age $\geq 75$ years (doubled), diabetes mellitus, prior stroke or transient ischemic attack or thromboembolism (doubled), vascular disease, age 65 to 74 years, sex category (female), CVTE cerebrovascular thromboembolism, LA left atrial, LAA left atrial appendage, LV left ventricular, $L V E F$ left ventricular ejection fraction, NOAF new-onset supraventricular arrhythmia, TEE transesophageal echocardiography, VA ECMO veno-arterial extracorporeal membrane oxygenation

${ }^{a}$ Including LA/LAA SEC and low LAA flow velocity

${ }^{b}$ Revealed at a supplementary TEE performed 17 days after NOAF onset because of ischemic stroke

${ }^{c}$ Revealed at the second TEE study performed in all patients with arterial thromboembolic event except patient 4

${ }^{\mathrm{d}}$ Arterial thromboembolic mechanism was assessed by an independent committee

e Causative subtype of ischemic stroke (large artery atherosclerosis, cardio-aortic embolism, small-artery occlusion, other cause, or unknown cause) was determined using the Stop Stroke Study Trial of ORG 10172 in Acute Stroke Treatment classification criteria [27]

${ }^{f}$ Among patients with non-cerebrovascular thromboembolism and those with evident cardio-aortic embolism as a causative subtype of ischemic stroke

Table 4 Univariate and multivariable analyses of factors associated with cardiovascular events ${ }^{\mathrm{a}}$

\begin{tabular}{|c|c|c|c|c|}
\hline \multirow[t]{2}{*}{ Variable } & \multicolumn{2}{|c|}{ Univariate analysis } & \multicolumn{2}{|c|}{ Multivariable analysis } \\
\hline & $\mathrm{HR}(95 \% \mathrm{Cl})$ & $p$ value & $\mathrm{HR}(95 \% \mathrm{Cl})$ & $p$ value \\
\hline Septic shock ${ }^{b}$ & $2.72(1.30-5.69)$ & 0.01 & $2.36(1.06-5.29)$ & 0.04 \\
\hline Electrical cardioversion attempt ${ }^{b}$ & $2.33(1.17-4.65)$ & 0.02 & $1.51(0.72-3.17)$ & 0.27 \\
\hline LV systolic dysfunction, d & $2.75(1.48-5.08)$ & 0.001 & $2.06(1.05-4.05)$ & 0.03 \\
\hline LA/LAA dysfunction ${ }^{\text {d, e }}$ & $1.14(0.54-2.39)$ & 0.73 & $0.85(0.39-1.87)$ & 0.69 \\
\hline Severe aortic atheroma ${ }^{d}$ & $0.58(0.26-1.33)$ & 0.20 & $0.60(0.26-1.40)$ & 0.24 \\
\hline
\end{tabular}

$C l$ confidence interval, $H R$ hazard ratio, $L A$ left atrial, $L A A$ left atrial appendage, $L V$ left ventricular

${ }^{a}$ Composite of arterial thromboembolic events, major bleeding events, and death from any cause

${ }^{\mathrm{b}}$ On the first day of new-onset atrial fibrillation onset

' LV ejection fraction $\leq 45 \%$

${ }^{\mathrm{d}}$ At the first echocardiography

e LA/LAA dense spontaneous echo contrast or LAA low velocity 
the only TEE parameters associated with major bleeding events (Table 2).

\section{8-day mortality}

Baseline clinical characteristics, initial severity and NOAF management were similar between survivors and non-survivors, with the exception of the Simplified Acute Physiology Score II score on ICU admission, which was associated with mortality (Table 1 ). LV ejection fraction was the only TEE parameter associated with mortality (Table 2).

\section{Discussion}

Our study aimed at describing the early TEE abnormalities in critically ill mechanically ventilated patients with sepsis and NOAF, and at estimating the cardiovascular risk by performing a systematic comprehensive morphological work-up with TEE. We found that LV systolic dysfunction, severe aortic atheroma, and LA/LAA dysfunction were common, and that the incidence of cardiovascular events was very high, including arterial thromboembolic event (7\%), major bleeding event (19\%), and all-cause death (29\%). Among the initial TEE abnormalities, only LV systolic dysfunction was independently associated with a poor prognosis.

\section{Thrombotic and bleeding risks}

In line with our results, Yoshida et al. reported a 4.6\% incidence of stroke in ICU patients with NOAF [28]. Walkey et al. showed that septic patients with NOAF had a greater risk of in-hospital stroke, as compared with their counterparts [2]. We also report that major bleeding events, most of which were life-threatening, were almost three times more frequent than thromboembolic events (19\% versus $7 \%$ ). Similarly, Walkey et al. showed a $12.6 \%$ incidence of bleeding in a large retrospective cohort of patients with sepsis and NOAF [29]. Gastrointestinal bleedings in our cohort of patients were more common and severe than in previous cohort in critically ill patients [30]. Krag et al. reported a $2.4 \%$ incidence of clinically important gastrointestinal bleeding in 1034 critically ill patients, which none of them was fatal [30]. Upper gastrointestinal lesions commonly seen in ICU patients requiring mechanical ventilation [31] may also increase bleeding risk among patients with sepsis who are receiving therapeutic anticoagulation. Therefore, thrombotic and bleeding risks in patients with sepsis and NOAF seem to be higher than those reported in cardiology wards. In a recent clinical trial studying early versus delayed cardioversion in patients with NOAF in the Emergency Department, Pluymaerkers et al. reported a $0.5 \%$ incidence of arterial thromboembolic events and no major bleeding events within 4 weeks of follow-up [32].
The underlying mechanisms of the cardiovascular events are complex in this context. NOAF may be a marker of greater sepsis severity, associated with an increased risk of thrombotic and bleeding events through hemodynamic collapse, increased systemic inflammation, and coagulopathy [33, 34]. Sepsis could predispose to LA/LAA thrombus formation in patients with NOAF. Virchow's triad-including abnormal blood stasis, hypercoagulable state, and endothelial dysfunction-could be exacerbated in this context $[35,36]$. In this setting, significant left-sided valve disease, associated with the occurrence of thrombotic events in our cohort, could be a potential etiologic factor in the development of them. In addition, due to inclusion criteria some of patients may have unrecognized pre-existing paroxysmal AF. At last, patients hospitalized in the ICU for sepsis and requiring mechanical ventilation seem to have a high baseline thrombotic and bleeding risks, irrespective of NOAF, as shown in our cohort of elderly patients with frequent comorbidities such as cardiovascular disease and arterial hypertension.

\section{Therapeutic anticoagulation}

Observational data suggested that therapeutic anticoagulation was not associated with a reduced risk of ischemic stroke, but was associated with a higher bleeding risk among propensity score-matched patients with sepsis and atrial fibrillation [29]. However, there are no robust data on the net clinical benefit of therapeutic anticoagulation in patients with sepsis and NOAF. In line with our results, Yoshida et al. reported that $40 \%$ of critically ill patients with NOAF received therapeutic anticoagulation [28]. In patients with septic shock eligible for early NOAF cardioversion $(<24 \mathrm{~h})$, a recent French survey has shown that $22 \%$ of intensivists initiated immediate therapeutic anticoagulation, whereas $27 \%$ initiated therapeutic anticoagulation after $24 \mathrm{~h}$, and $44 \%$ after $48 \mathrm{~h}$ of sustained NOAF [37]. Similarly, we reported that only one-third of patients received therapeutic anticoagulation prior to electric shock. These deviations from recent European guidelines that recommend therapeutic anticoagulation before any emergent cardioversion $[4,10]$ confirm that the cardiologic approach does not seem appropriate in septic patient at high risk of bleeding. In the light of our results, specific therapeutic for NOAF in patients with sepsis such as electric shock and therapeutic anticoagulation should be used with caution. Hence, identifying patients with sepsis and NOAF most likely to benefit from therapeutic anticoagulation is a major clinical challenge. We found that the $\mathrm{CHA}_{2} \mathrm{DS}_{2}$-VASc and HAS-BLED scores, used for clinical decision-making on anticoagulation in the cardiology ward, were not associated with any of the cardiovascular events. 
Similarly, Walkey et al. reported a poor performance of $\mathrm{CHA}_{2} \mathrm{DS}_{2}$-VASc scores to stratify risk of stroke during sepsis in a large retrospective cohort of patients with sepsis and atrial fibrillation [29]. Finally, our results confirm the need for bedside cardiovascular risk estimation tools, to help decisions to be made about therapeutic anticoagulation and its timing.

\section{TEE for cardiovascular risk estimation}

A TEE-guided strategy for estimating cardiovascular risk may be questionable. Although initial TEE revealed frequent LAA dysfunction (including dense SEC or low velocity), severe aortic atheroma, and LV systolic dysfunction, only the last of these was associated with a poor prognosis. Moreover, a systematic early TEE followed by a second TEE in two-thirds of patients revealed only one LAA thrombus. In line with our results, Seemann et al. reported myocardial dysfunction in $40 \%$ of patients with septic shock and NOAF [38]. LV systolic dysfunction and NOAF may be two components of myocardial septic dysfunction. The clinical and prognosis significances of LV systolic dysfunction during sepsis are a matter of debate [39]; however, our findings suggest that its association with NOAF is associated with a poor prognosis. Two previous studies reported the prevalence of thrombus, dense SEC and severe aortic atheroma in ambulatory cardiological patients undergoing TEE for NOAF cardioversion [40, 41]. In comparison with our results, Kleemann et al. reported a similar prevalence of thrombus (1\%), dense SEC (9.5\%), and aortic atheroma (21\%), whereas Stoddart et al. reported a higher prevalence of thrombus (14\%) and dense SEC (39\%) [40, 41]. Those differences may be explained by the higher number of patients with structural heart disease in the study by Stoddart et al. (94\%), as compared with the study by Kleemann et al. (49\%) and our study (18\%).

\section{Study strengths and limitations}

This multicenter study was conducted in 10 tertiary university ICUs, where TEE is routinely used in mechanically ventilated critically ill patients. The major strengths of our study are: (i) the comprehensive search for risk factors for cardiovascular events, including systematic morphological work-up with two sequential TEE studies (initial TEE to identify patients at high risk of cardiovascular events, and the second TEE to investigate LA/LAA thrombus formation); (ii) its prospective design with adjudication of arterial thromboembolic events and major bleeding events; and (iii) the mean level of inclusion of 0.5 patient per month per center seems to be an appropriate number with regard to the eligibility criteria and is consistent with consecutive and exhaustive recruitment. Our study has several limitations. First, the relatively small number of patients limited power in all analyses. This may explain the absence of association between TEE abnormalities and outcomes. One could also object that arterial thromboembolic event might have been more relevant as a primary outcome, yet we did not consider this option, because of its low incidence. Instead, we used a composite outcome that reflects the net clinical benefit of therapeutic anticoagulation. Second, the incidence of arterial thromboembolic events may have been underestimated due to the fact that (i) the second TEE was not performed in all patients; (ii) TEEs performed in the first few days after NOAF onset may not capture LA/LAA thrombus formation within this time frame and (iii) cerebral magnetic resonance imaging was not systematic. Third, the present study was an observational study with potential indication bias. Rhythm/ rate control and therapeutic anticoagulation could have influenced the occurrence of thrombotic and bleeding events. Fourth, we excluded patients who had a history of atrial fibrillation, in whom long-term cardiovascular risk stratification has been well studied [10]. Fifth, although an independent committee assessed arterial thromboembolic mechanism using a previous published classification, make a clear distinction between the "cardio-aortic embolism" and sepsis-related hypotension as a cause of stroke remains difficult. Finally, as this study was conducted in France, our findings may not be applicable elsewhere; however, the incidences of cardiovascular events reported in other nations $[2,3$, $28,29]$ were similar to our findings.

\section{Conclusions}

TEE abnormalities (including LA/LAA dysfunction, severe aortic atheroma, and LV dysfunction), and cardiovascular events were common in critically ill patients with sepsis and NOAF. However, only LV systolic dysfunction was independently associated with cardiovascular events. Consequently, transesophageal echocardiography appears to be limited in this context for estimating cardiovascular risk, albeit the sample size was relatively small.

\footnotetext{
Abbreviations

$\mathrm{CHA}_{2} \mathrm{DS}_{2}$-VASc: Congestive heart failure hypertension, hypertension, age $\geq 75$ years (doubled), diabetes mellitus, prior stroke or transient ischemic attack or thromboembolism (doubled), vascular disease, age 65 to 74 years, sex category (female); HAS-BLED: Hypertension abnormal renal/liver function, stroke, bleeding history or predisposition, labile international normalized ratio, elderly, drugs/alcohol concomitantly; ICU: Intensive care unit; IQR: Interquartile range; LA: Left atrial; LAA: Left atrial appendage; LV: Left ventricular; NOAF: New-onset atrial fibrillation; SEC: Spontaneous echo contrast; TEE: Transesophageal echocardiography.
} 


\section{Supplementary Information}

The online version contains supplementary material available at https://doi. org/10.1186/s13613-021-00934-1.

Additional file 1: Table S1. Definition of CHA2DS2-VASC and HASBLED risk scores, arterial thromboembolic events, and bleeding events. Table S2. Stop Stroke Study Trial of Org 10172 in Acute Stroke Treatment (SSS-TOAST) Classification Criteria to Determine Causative Subtypes of Acute Ischemic Stroke. Figure S1. The decision algorithm to assign a mechanism using the Stop Stroke Study Trial of Org 10172 in Acute Stroke Treatment (SSS-TOAST) Classification Criteria. Table S3. Initial transthoracic and transesophageal echocardiographic variables according to 28-day cardiovascular events. Table S4. New onset atrial fibrillation and sepsis management during 28-day follow-up in intensive care unit. Table S5. Description of 28-day ISTH major bleeding events. Table S6. Baseline clinical characteristics at intensive care unit admission, initia severity and new-onset atrial fibrillation management according to 28-day cardiovascular events. Table S7. Multivariate analyses of factors associated with cardiovascular events including baseline clinical characteristics (SAPSII score, CHADS2Vasc2 and HAS-BLED) and antithrombotic management (antiplatelet therapy and therapeutic anticoagulation on the day of NOAF onset).

\section{Acknowledgements}

The authors thank Nadjib Hammoudi, Mathieu Schmidt, and Nicolas Weiss for being part of the independent committee who adjudicated clinical events; and the FAST study group: Bertrand Guidet, Hafid Ait-Oufella, and Simon Bourcier (Médecine Intensive Réanimation, Hôpital Saint-Antoine, Paris, France); Daniel Da Silva (Médecine Intensive Réanimation, Centre Hospitalier de Saint-Denis, Saint Denis, France); Sebastien Jochmans (Médecine Intensive Réanimation, Centre Hospitalier Melun, Melun, France); Jean Dellamonica (Médecine Intensive Réanimation, Centre Hospitalier Universitaire de Nice, Nice, France); Jean-François Timsit (Department of Intensive Care Medicine and Infectious Diseases, Bichat-Claude Bernard Hospital, Paris, France); Bruno Megarbane (Service de Réanimation Médicale et Toxicologique, Hôpital Lariboisière, Paris, France); and Jean-Luc Diehl (Médecine Intensive Réanimation, Hôpital Européen Georges-Pompidou, Paris, France). Editorial support was provided by Sophie Rushton-Smith (MedLink Healthcare Communications), and was funded by the authors.

\section{Authors' contributions}

VL, SE, AC, and MF contributed to study conception and design. VL, SE, JJ, KR, $\mathrm{LL}, \mathrm{OS}, \mathrm{SV}, \mathrm{FC}, \mathrm{NA}, \mathrm{RS}$, and $\mathrm{DD}$ participated in acquiring the data. VL, SE, AC analyzed off-line echocardiographic studies. VL, SE, NL, FC, GV, AMD, AC and MF analyzed and interpreted the study data. VL and MF drafted the original manuscript. All authors revised the manuscript for important intellectual content. All authors read and approved the final manuscript.

\section{Funding}

No source of funding.

\section{Availability of data and materials}

All data and materials are fully complying with field standards and might be available after request.

\section{Declarations}

\section{Ethics approval and consent to participate}

The study has been approved by the Comité de Protection des Personnes Ilede-France 5 (ref. 14941) as a component of standard care and patient consent was waived, as per French Law. Written and oral information about the study was given to the patient or next of kin.

\section{Consent for publication}

Not applicable.

\section{Competing interests}

The authors declare that they have no competing interests related to the present study.

\section{Author details}

'Sorbonne Université, Assistance Publique-Hôpitaux de Paris (AP-HP), Service de Médecine Intensive Réanimation, Département Médico-Universitaire APPROCHES, Hôpital Tenon, 4 rue de la Chine, 75020 Paris, France. ${ }^{2}$ Université Paris Est, Groupe de Recherche Clinique GR05 CARMAS, Institut Mondor de recherche biomédicale, INSERM, Créteil, France. ${ }^{3}$ Department of Cardiology, UNICO Cardio-Oncology Program, Hôpital Saint-Antoine, AP-HP, Paris, France. ${ }^{4}$ INSERM U 856, Paris, France. ${ }^{5}$ Sorbonne Université, INSERM, Institut Pierre Louis d'Epidémiologie et de Santé Publique IPLESP, AP-HP, Paris, France. ${ }^{6}$ Sorbonne Université, Public Health Department, Saint Antoine Hospital, AP-HP, Paris, France. ${ }^{7}$ Service de Médecine Intensive Réanimation, Hôpital Saint-Antoine, AP-HP, Sorbonne Université, Paris, France. ${ }^{8}$ Service de Médecine Intensive Réanimation, Département Médico-Universitaire Médecine, Hôpitaux Universitaires Henri Mondor-Albert Chenevier, AP-HP, Créteil, France. ${ }^{9}$ Service de Médecine Intensive Réanimation, Centre Hospitalier de Saint-Denis, Saint Denis, France. ${ }^{10}$ Service de Médecine Intensive Réanimation, Groupe Hospitalier Sud Ile-de-France, Centre Hospitalier Melun, Melun, France. ${ }^{11}$ Service de Réanimation Médicale et Toxicologique, Hôpital Lariboisière, AP-HP, INSERM UMRS-1144, Université de Paris, Paris, France. ${ }^{12}$ Service de Médecine Intensive Réanimation, Gustave Roussy, Villejuif, France. ${ }^{13}$ Service de Médecine Intensive Réanimation, Hôpital Européen Georges-Pompidou, AP-HP, Université Paris-Descartes, Paris, France. ${ }^{14}$ Department of Intensive Care Medicine and Infectious Diseases, Bichat-Claude Bernard University Hospital, AP-HP, Paris, France. ${ }^{15}$ Service de Médecine Intensive Réanimation, Hôpital I'Archet 1, Centre Hospitalier Universitaire de Nice, and UR2CA Unité de Recherche Clinique Côte d'Azur, Université Côte d'Azur, Nice, France. ${ }^{16}$ UMR-S ICAN 1166, Sorbonne Université, Paris, France.

Received: 28 June 2021 Accepted: 2 October 2021

Published online: 18 October 2021

\section{References}

1. Klein Klouwenberg PMC, Frencken JF, Kuipers S, Ong DSY, Peelen LM, van Vught LA, et al. Incidence, predictors, and outcomes of new-onset atrial fibrillation in critically ill patients with sepsis. A cohort study. Am J Respir Crit Care Med. 2017;195:205-11.

2. Walkey AJ, Wiener RS, Ghobrial JM, Curtis LH, Benjamin EJ. Incident stroke and mortality associated with new-onset atrial fibrillation in patients hospitalized with severe sepsis. JAMA. 2011;306:2248-54.

3. Champion S, Lefort $Y$, Gaüzère B-A, Drouet D, Bouchet BJ, Bossard G, et al. CHADS2 and CHA2DS2-VASc scores can predict thromboembolic events after supraventricular arrhythmia in the critically ill patients. J Crit Care. 2014;29:854-8.

4. January CT, Wann LS, Calkins H, Chen LY, Cigarroa JE, Cleveland JC, et al. 2019 AHA/ACC/HRS focused update of the 2014 AHA/ACC/HRS guideline for the management of patients with atrial fibrillation: a report of the American College of Cardiology/American Heart Association Task Force on clinical practice guidelines and the heart rhythm society in collaboration with the society of thoracic surgeons. Circulation. 2019;140:e125-51.

5. Zabalgoitia M, Halperin JL, Pearce LA, Blackshear JL, Asinger RW, Hart RG. Transesophageal echocardiographic correlates of clinical risk of thromboembolism in nonvalvular atrial fibrillation. Stroke prevention in atrial fibrillation III investigators. J Am Coll Cardiol. 1998;31:1622-6.

6. Asinger RW, Koehler J, Pearce LA, Zabalgoitia M, Blackshear JL, Fenster PE, et al. Pathophysiologic correlates of thromboembolism in nonvalvular atrial fibrillation: II. Dense spontaneous echocardiographic contrast (the stroke prevention in atrial fibrillation [SPAF-III] study). J Am Soc Echocardiogr. 1999;12:1088-96.

7. Fatkin D, Kelly RP, Feneley MP. Relations between left atrial appendage blood flow velocity, spontaneous echocardiographic contrast and thromboembolic risk in vivo. J Am Coll Cardiol. 1994;23:961-9.

8. Pollick C, Taylor D. Assessment of left atrial appendage function by transesophageal echocardiography. Implications for the development of thrombus. Circulation. 1991;84:223-31. 
9. Di Biase L, Natale A, Romero J. Thrombogenic and arrhythmogenic roles of the left atrial appendage in atrial fibrillation. Circulation. 2018;138:2036-50.

10. Hindricks G, Potpara T, Dagres N, Arbelo E, Bax JJ, Blomström-Lundqvist C, et al. 2020 ESC guidelines for the diagnosis and management of atrial fibrillation developed in collaboration with the European Association for Cardio-Thoracic Surgery (EACTS): the Task Force for the diagnosis and management of atrial fibrillation of the European Society of Cardiology (ESC) developed with the special contribution of the European Heart Rhythm Association (EHRA) of the ESC. Eur Heart J. 2021;42:373-498.

11. Vieillard-Baron A, Boyd J. Non-antiarrhythmic interventions in new onset and paroxysmal sepsis-related atrial fibrillation. Intensive Care Med. 2018:44:94-7.

12. Singer M, Deutschman CS, Seymour CW, Shankar-Hari M, Annane D, Bauer $M$, et al. The third international consensus definitions for sepsis and septic shock (sepsis-3). JAMA. 2016;315:801-10.

13. Zipes D. Specific arrhythmias: diagnosis and treatment. In: Braunwald E, editor. Heart disease: a textbook of cardiovascular medicine. Philadelphia: Elsevier; 1992. p. 667-725.

14. Camm AJ, Lip GYH, De Caterina R, Savelieva I, Atar D, Hohnloser SH, et al. 2012 focused update of the ESC guidelines for the management of atrial fibrillation: an update of the 2010 ESC guidelines for the management of atrial fibrillation. Developed with the special contribution of the European Heart Rhythm Association. Eur Heart J. 2012;33:2719-47.

15. Slama M. Echocardiographie Doppler chez les patients en état critique: un outil de monitorage aux multiples modalités. In: Chabert M, editor. Echocardiogr Doppler Chez Patients En État Critique. Issy les moulineaux: Elsevier Masson; 2008. p. 9-24.

16. Le Gall JR, Lemeshow S, Saulnier F. A new simplified acute physiology score (SAPS II) based on a European/North American multicenter study. JAMA. 1993:270:2957-63.

17. Vincent JL, Moreno R, Takala J, Willatts S, De Mendonça A, Bruining H, et al. The SOFA (sepsis-related organ failure assessment) score to describe organ dysfunction/failure. On behalf of the working group on sepsisrelated problems of the European Society of Intensive Care Medicine. Intensive Care Med. 1996:22:707-10.

18. Expert Round Table on Echocardiography in ICU. International consensus statement on training standards for advanced critical care echocardiography. Intensive Care Med. 2014;40:654-66.

19. Lang RM, Badano LP, Mor-Avi V, Afilalo J, Armstrong A, Ernande L, et al. Recommendations for cardiac chamber quantification by echocardiography in adults: an update from the American Society of Echocardiography and the European Association of Cardiovascular Imaging. Eur Heart J Cardiovasc Imaging. 2015;16:233-71.

20. Amarenco P, Cohen A, Tzourio C, Bertrand B, Hommel M, Besson G, et al. Atherosclerotic disease of the aortic arch and the risk of ischemic stroke. N Engl J Med. 1994:331:1474-9.

21. Di Angelantonio E, Ederhy S, Benyounes N, Janower S, Boccara F, Cohen A. Comparison of transesophageal echocardiographic identification of embolic risk markers in patients with lone versus non-lone atrial fibrillation. Am J Cardiol. 2005;95:592-6.

22. Montgomery DH, Ververis JJ, McGorisk G, Frohwein S, Martin RP, Taylor WR. Natural history of severe atheromatous disease of the thoracic aorta: a transesophageal echocardiographic study. J Am Coll Cardiol. 1996:27:95-101.

23. Mügge A, Kühn H, Nikutta P, Grote J, Lopez JA, Daniel WG. Assessment of left atrial appendage function by biplane transesophageal echocardiography in patients with nonrheumatic atrial fibrillation: identification of a subgroup of patients at increased embolic risk. J Am Coll Cardiol. 1994:23:599-607.

24. Tunick PA, Kronzon I. Atheromas of the thoracic aorta: clinical and therapeutic update. J Am Coll Cardiol. 2000;35:545-54

25. Connolly SJ, Ezekowitz MD, Yusuf S, Eikelboom J, Oldgren J, Parekh A, et al. Dabigatran versus warfarin in patients with atrial fibrillation. N Engl J Med. 2009;361:1139-51.

26. Schulman S, Kearon C, Subcommittee on Control of Anticoagulation of the Scientific and Standardization Committee of the International Society on Thrombosis and Haemostasis. Definition of major bleeding in clinical investigations of antihemostatic medicinal products in non-surgical patients. J Thromb Haemost. 2005;3:692-4
27. Ay H, Furie KL, Singhal A, Smith WS, Sorensen AG, Koroshetz WJ. An evidence-based causative classification system for acute ischemic stroke. Ann Neurol. 2005;58:688-97.

28. Yoshida T, Uchino S, Sasabuchi Y, Hagiwara Y, AFTER-ICU study group. Prognostic impact of sustained new-onset atrial fibrillation in critically ill patients. Intensive Care Med. 2020;46:27-35.

29. Walkey AJ, Evans SR, Winter MR, Benjamin EJ. Practice patterns and outcomes of treatments for atrial fibrillation during sepsis: a propensitymatched cohort study. Chest. 2016;149:74-83.

30. Krag M, Perner A, Wetterslev J, Wise MP, Borthwick M, Bendel S, et al. Prevalence and outcome of gastrointestinal bleeding and use of acid suppressants in acutely ill adult intensive care patients. Intensive Care Med. 2015:41:833-45.

31. van der Voort PH, van der Hulst RW, Zandstra DF, Geraedts AA, van der Ende A, Tytgat GN. Prevalence of Helicobacter pylori infection in stressinduced gastric mucosal injury. Intensive Care Med. 2001;27:68-73.

32. Pluymaekers NAHA, Dudink EAMP, Luermans JGLM, Meeder JG, Lenderink T, Widdershoven J, et al. Early or delayed cardioversion in recent-onset atrial fibrillation. N Engl J Med. 2019;380:1499-508.

33. Kreutz RP, Bliden KP, Tantry US, Gurbel PA. Viral respiratory tract infections increase platelet reactivity and activation: an explanation for the higher rates of myocardial infarction and stroke during viral illness. J Thromb Haemost. 2005;3:2108-9.

34. Zeerleder S, Hack CE, Wuillemin WA. Disseminated intravascular coagulation in sepsis. Chest. 2005;128:2864-75.

35. Semeraro N, Ammollo CT, Semeraro F, Colucci M. Coagulopathy of acute sepsis. Semin Thromb Hemost. 2015;41:650-8.

36. Watson T, Shantsila E, Lip GYH. Mechanisms of thrombogenesis in atrial fibrillation: Virchow's triad revisited. Lancet. 2009;373:155-66.

37. Labbé V, Bagate F, Cohen A, Voiriot G, Fartoukh M, Mekontso-Dessap A. A survey on the management of new onset atrial fibrillation in critically ill patients with septic shock. J Crit Care. 2020;61:18-20.

38. Seemann A, Boissier F, Razazi K, Carteaux G, de Prost N, Brun-Buisson C, et al. New-onset supraventricular arrhythmia during septic shock: prevalence, risk factors and prognosis. Ann Intensive Care. 2015;5:27.

39. Aneman A, Vieillard-Baron A. Cardiac dysfunction in sepsis. Intensive Care Med. 2016;42:2073-6.

40. Kleemann T, Becker T, Strauss M, Schneider S, Seidl K. Prevalence of left atrial thrombus and dense spontaneous echo contrast in patients with short-term atrial fibrillation < 48 hours undergoing cardioversion: value of transesophageal echocardiography to guide cardioversion. J Am Soc Echocardiogr. 2009;22:1403-8.

41. Stoddard MF, Dawkins PR, Prince CR, Ammash NM. Left atrial appendage thrombus is not uncommon in patients with acute atrial fibrillation and a recent embolic event: a transesophageal echocardiographic study. J Am Coll Cardiol. 1995:25:452-9.

\section{Publisher's Note}

Springer Nature remains neutral with regard to jurisdictional claims in published maps and institutional affiliations.

\section{Submit your manuscript to a SpringerOpen ${ }^{\circ}$ journal and benefit from:}

- Convenient online submission

- Rigorous peer review

- Open access: articles freely available online

- High visibility within the field

Retaining the copyright to your article

Submit your next manuscript at springeropen.com 